\title{
Effects of Taper Ratio on Aircraft Wing Aerodynamic Parameters: A Comperative Study
}

\author{
İbrahim Halil Güzelbey' ${ }^{1}$ Yüksel Eraslan²*, Mehmet Hanifi Doğru ${ }^{3}$ \\ 1,2Aircraft and Aerospace Engineering Department/Gaziantep University, Turkey \\ ${ }_{3}^{3}$ Pilotage Department/Gaziantep University, Turkey \\ I.H. Güzelbey (0000-0002-4235-9746), Y. Eraslan (0000-0002-5158-5171), M.H.Doğru (0000-0001-6038-8308)
}

\begin{abstract}
Wing design is one of the most important tasks for a designer to overcome during an aircraft design process. Therefore, a designer need to optimize so many wing geometrical parameters with the aim of obtaining an efficient wing geometry complying with requirements of the design. Taper ratio is one of these parameters, which is the ratio of root and tip chord lengths of a wing. In this study, firstly, a high aspect ratio rectangular aircraft wing was numerically investigated in terms of some aerodynamic parameters including induced drag coefficient, Oswald efficiency factor and lift coefficient together with its span-wise distribution by means of XFLR5 computational fluid dynamics program. The assessment of mesh accuracy of the program was done at the beginning of the analyses. Later on, with the aim of observing the effects of taper ratio on aircraft wing aerodynamic parameters, the revised versions of the wing, which have the taper ratios from 0.2 to 1.2 (with the increment of 0.2) were analyzed. In conclusion, depending on the analyses results, the wings having different taper ratios were compared in terms of obtained aerodynamic parameters and span-wise lift distributions. Moreover, tip vortices of each wing, together with their sizes, were obtained and also compared.
\end{abstract}

Keywords: Aircraft wing design, taper ratio, lift distribution, induced drag coefficient.

\section{INTRODUCTION}

An aircraft designer has so many tasks to overcome during an aircraft design process. One of the most important tasks is to design an efficient wing complying with the determined requirements. This is generally possible with optimizing so many geometrical and aerodynamic parameters of the wing $[1]$.

Geometrically, airfoil and planform geometries are the main terms to define a wing. An aerodynamically efficient wing can be designed when the suitable airfoil(s) and planform geometry coupled. Therefore, planform geometry is one of the most important geometries during an aircraft design process. Taper ratio is one of the parameters on planform geometry which means the ratio of the root and tip chord lengths of a wing. Hence, its effects on wing's aerodynamic parameters are also important and should be taken into consideration during a wing design process [2] [3].

The effects of the taper ratio on wing aerodynamic parameters can be obtained by means of numerical or experimental analyses [4]. At the conceptual design phase of an aircraft, it can be preferable to use computational fluid dynamics programs rather than time consuming experimental setups. There are so many programs to perform these analyses such as Ansys Fluent, XFLR5 and Solidworks Flow Simulation. In the literature, including numerical analyses, there are so many studies about investigating the aerodynamic parameters of aircraft wings.

Zhang et al. [5] performed experimental analysis at low-speed wind tunnel of Beijing University of Aeronautics and Astronautics with the aim of investigation of aerodynamic performance effects of taper ratio on delta wings. The study includes the analysis of the delta wing models at $3.45 \times 10^{5}$ Reynolds number and geometrically having taper ratios (0$0.79)$ varying with different aspect ratios. For the analysis, there were 16 cropped nonslender delta wing models with a 40 deg swept leading edge having root chord length of $200 \mathrm{~mm}$ used in this study. The analysis results show that the wings having taper ratios lower than 0.3 have constant lift coefficients. Also it was found that the delta wings having the taper ratio between 0.3 to 0.68 , stall angle and maximum lift increases with taper ratio and reaches their maximum 
values at 0.725 taper ratio. In addition, the wings of taper ratio lower than 0.5 found to have constant potential lift factor $\left(\mathrm{K}_{\mathrm{p}}=2.3\right)$ and induced-drag factors $(\mathrm{K}=0.3)$. When taper ratio is larger than $0.5, \mathrm{~K}_{\mathrm{p}}$ found to be decrease quickly with taper ratio increase.

Moreover, this change was grouped in two aspect ratio scenarios of aspect ratios from 0.5 to 2.38 and 0.56 to 4.77 . With respect to anaylsis results, at the taper ratio from 0 to 0.3 , drag of all cropped delta wings at low angles of attack were found to be less than noncropped ones. At taper ratios from 0.3 to 0.79 , it was found that the stall angle of the wing will be delayed with the taper ratio and maximum lift coefficient increases.

Bravo-Mosquera et al. [6] presented conceptual design and prototype of an agricultural aircraft. Following the traditional design methods applied, six different winglet designs, which have different cant angles analyzed by means of a computational fluid dynamics program using ReynoldsAveraged-Navier-Stokes (RANS) equations. The aim of the analyses was determining the winglet design providing the best aerodynamic characteristics. The results shown that the configuration including winglet cant angles of $45^{\circ}, 15^{\circ}$ and $-15^{0}$ have maximum lift coefficient of approximately 1.7 at $18^{\circ}$ angle of attack. Moreover, among the other configurations, this configuration was also found to have maximum aerodynamic efficiency with L/D ratio of 22.91 at $2^{0}$ angle of attack. For range factor of , configuration 2 (with $-15^{0}$,$30^{\circ},-45^{\circ}$ cant angled winglets) found to have best performance with 29.35. Later on, these analyses were expanded to complete aircraft and obtained lift, drag and pitching moment coefficients were investigated together with wingtip vortex structures. At the end of the study, they obtained that multi winglet devices were contributing on improving performance of the aircraft, providing control on the sprayed product, reducing the induced drag and bending moment of the wing.

Qin et al. [7] performed computational fluid dynamics analyses using Reynolds-averaged Navier-Stokes (RANS) equations on a baseline blended wing body configuration with the aim of obtaining the effects of span-wise lift distribution. After the grid sensitivity study on total drag, they obtained the main factor decreasing the aerodynamic performance of the baseline body is wing loading together with shock wave. They revised the body to three models having different span-wise lift distributions and investigated the change in aerodynamic performances. Total drag coefficients of baseline, elliptic, averaged and triangular models were found to be $0.0327,0.0284,0.0278$ and 0.0287 , respectively. In addition, the induced drag values at 0.3 mach and lift coefficient of 0.23 was found for each model as 0.00333 (baseline), 0.00268 (elliptic), 0.00325 (averaged) and 0.00470 (triangular).

Lee et al. [8] investigated the effect of winglet dihedral on a tapered and swept wing at a low Reynolds number of
$1.81 \times 10^{5}$. Experimental analyses of the winglets having different dihedral angles were performed at a wind tunnel in McGill University at $35 \mathrm{~m} / \mathrm{s}$ freestream velocity and 0.73 lift coefficient. Dihedral angles varied between -40 to 87.5 degrees. According to results of the analyses, it was obtained that the induced-drag of a wing always reduces with the use of a winglet and the winglet, which have negative dihedral, decreases lift-induced drag more than positive dihedral. Moreover, it was revealed that, the inner region of the tip vortex behaviors is similar for the wing with or without winglets. In the result, the total circulation to bound circulation ratio $\left(\Gamma_{\mathrm{o}} / \Gamma_{\mathrm{b}}\right)$ was found to be 0.4 and core circulation to total circulation ratio $\left(\Gamma_{\mathrm{c}} / \Gamma_{\mathrm{o}}\right)$ was found to be 0.63 for $\mathrm{x} /$ $\mathrm{c}_{\mathrm{r}}=2.75$ location.

In this study, firstly, a high aspect ratio rectangular aircraft wing was numerically investigated in terms of some aerodynamic parameters including induced drag coefficient, Oswald efficiency factor, and lift coefficient together with span-wise lift distribution by means of XFLR5 computational fluid dynamics program. The assessment of mesh accuracy of the program was done at the beginning of the analyses. Later on, with the aim of observing the effects of taper ratio on aircraft wing aerodynamic parameters, the revised versions of the wing, which have the taper ratios from 0.2 to 1.2 (with the increment of 0.2 ) were analyzed. In conclusion, depending on the analyses results, the wings having different taper ratios were compared in terms of obtained aerodynamic parameters and span-wise lift distributions. Moreover, tip vortices of each wing, together with their sizes, were obtained and also compared.

\section{MATERIAL AND METHOD}

\subsection{Planform Geometry and Aerodynamic Parameters of an Aircraft Wing}

Planform geometry is the top-view shape of a wing and effective on wing aerodynamic performance [9] [10]. Therefore, the geometrical parameters of a wing planform geometry are also important for a wing design. Changing these geometrical parameters properly with respect to their effects on the wing aerodynamic parameters can provide an improved aerodynamic performance to wing. Taper ratio $(\lambda)$, as a part of the wing planform geometry, is one of these important parameters to take into consideration during an aircraft wing design process. It is the ratio as stated in Equation (1), which is the ratio of the root $\left(c_{r}\right)$ and tip $\left(c_{t}\right)$ chord lengths as shown in Figure 1.

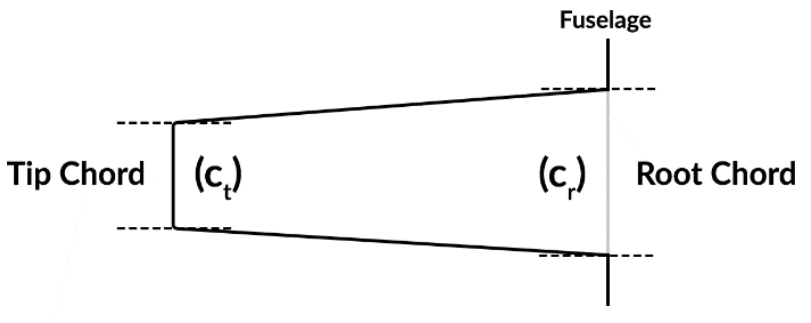

Figure 1: Aircraft wing root and tip chords 


$$
\lambda=\frac{c_{t}}{c_{r}}
$$

The main aerodynamic parameters of an aircraft wing are drag $\left(C_{D}\right)$, lift $\left(C_{L}\right)$ and pitching moment $\left(C_{M}\right)$ coefficients. The other aerodynamic performance parameters can be derived from these parameters, such as glide ratio, which is the ratio between lift coefficient and drag coefficients. Lift, drag and pitching moment coefficients can be calculated using the Equations (2) - (3) - (4). In these equations, A is the related area $\left(\mathrm{m}^{2}\right), \mathrm{V}$ is the freestream velocity $(\mathrm{m} / \mathrm{s}), F_{L}$ is the lift force $(\mathrm{N}), F_{D}$ is the drag force $(\mathrm{N}), \rho$ is the air density $(\mathrm{kg} /$ $\left.\mathrm{m}^{3}\right)$ and $\mathrm{M}$ is the moment $(\mathrm{Nm})$.

$$
\begin{aligned}
& C_{L}=\frac{2 F_{L}}{\rho \cdot A \cdot V^{2}} \\
& C_{D}=\frac{2 F_{D}}{\rho \cdot A \cdot V^{2}} \\
& C_{M}=\frac{2 M}{\rho \cdot A \cdot V^{2}}
\end{aligned}
$$

On the other hand, drag coefficient is divided into two components named as zero-lift drag coefficient $\left(C_{D_{o}}\right)$ and induced drag coefficient $\left(C_{D_{i}}\right)$ as shown in Equation (4). In the Equation (5), $\mathrm{AR}$ is the wing aspect ratio and $\mathrm{e}$ is the Oswald efficiency factor.

$$
\begin{aligned}
& C_{D}=C_{D_{o}}+C_{D_{i}} \\
& C_{D_{i}}=\frac{C_{L}^{2}}{A R \cdot \pi \cdot e}
\end{aligned}
$$

Oswald efficiency factor (e) is the value, which gives an idea about the similarity of a wing's span-wise lift distribution to the elliptical lift distribution. The elliptical lift distribution has Oswald efficiency factor of 1 and generally this is the maximum value of this parameter. There is another single parameter, which can represents wing efficiency in terms of induced drag, named as induced drag parameter $(\delta)$ and can be calculated from Equation (6) [11]. This parameter depends on only planform geometry and independent from angle of attack and lift coefficient.

$$
e=\frac{1}{1+\delta}
$$

\subsection{Numerical Analyses}

The main objective of this study is to investigate the effects of taper ratio on aerodynamic parameters of a wing design. In order to examine the effects, firstly, a high aspect ratio rectangular wing was modelled with a typical sailplane airfoil named as Wortmann FX 61-184 as a baseline model [12]. Later on, for the comparison, the baseline model's taper ratio was revised to $0.2,0.4,0.62,0.8$ and 1.2 by changing tip and root chord lengths while keeping wing area, aspect ratio and mean geometric chord (M.A.C.) values constant, as shown in Figure 2.

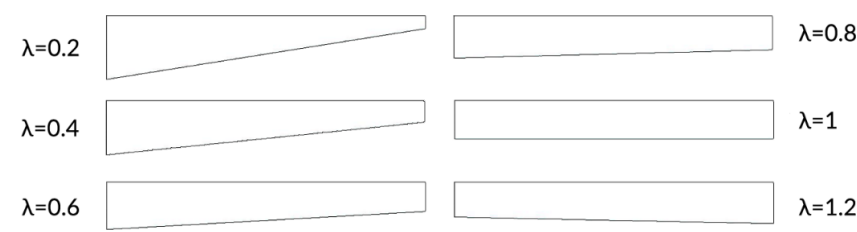

Figure 2: Planform geometries of revised wing models

Numerical analyses of the six different wing models were performed by XFLR5 program, which uses Vortex Lattice Method [13]. On the purpose of obtaining accurate results, before the analyses, the mesh accuracy of the program was done on the rectangular model for both drag and lift coefficients and results were shown in Figure 3. According to the results, all of the models were prepared to have number of mesh elements higher than 20000 to have accurate results independent from mesh. The mesh grid of the rectangular model is shown in Figure 4. The geometrical dimensions of the models, which were designed to have sufficient number of mesh elements, were given in Table 1.

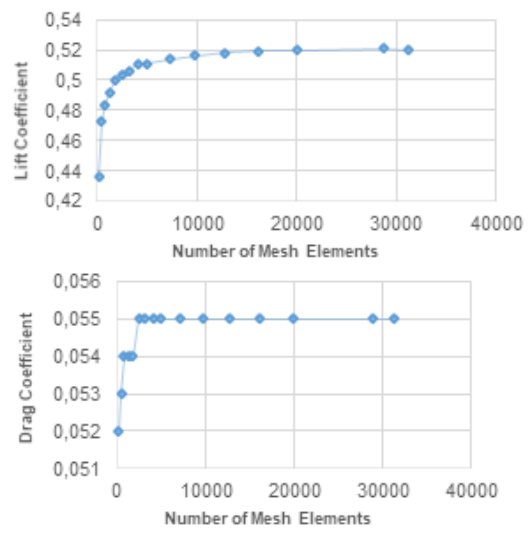

Figure 3: Baseline rectangular model mesh accuracy results

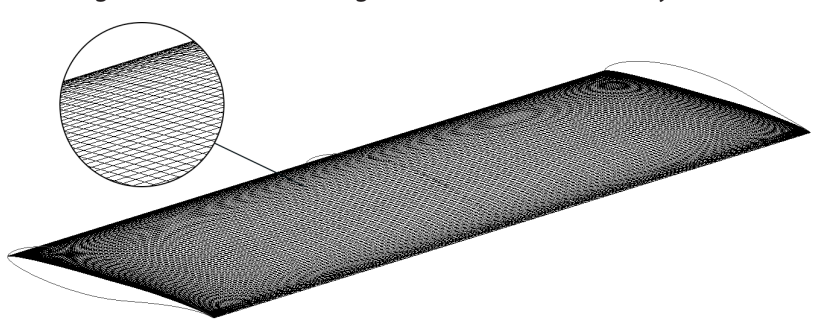

Figure 4: Mesh grid of the rectangular model with 20000 mesh elements

Table 1 Geometrical dimensions of the models

\begin{tabular}{|c|c|c|c|c|c|c|}
\hline Model & $\begin{array}{c}\text { Root Chord } \\
(\mathrm{m})\end{array}$ & $\begin{array}{c}\text { Tip Chord } \\
(\mathrm{m})\end{array}$ & $\begin{array}{c}\text { M.A.C. } \\
(\mathrm{m})\end{array}$ & $\begin{array}{c}\text { Wing Area } \\
\left(\mathrm{m}^{2}\right)\end{array}$ & $\begin{array}{c}\text { Aspect } \\
\text { Ratio }\end{array}$ & $\begin{array}{c}\text { Taper } \\
\text { Ratio }\end{array}$ \\
\hline 1 & 0.078 & 0.016 & & & & 0.2 \\
\hline 2 & 0.067 & 0.027 & & & & 0.4 \\
\hline 3 & 0.058 & 0.036 & \multirow{2}{*}{0.047} & & & \\
\hline 4 & 0.052 & 0.042 & & & 16.6 & 0.62 \\
\hline 5 & 0.047 & 0.047 & & & & 0.8 \\
\hline 6 & 0.043 & 0.051 & & & & 1 \\
\hline
\end{tabular}

Lastly, the models revised from the baseline rectangular wing model were numerically analyzed at $10^{6}$ Reynolds Number. As all the models have high aspect ratios, which is common on sailplane wing designs, the airspeed was used as $33.5 \mathrm{~m} / \mathrm{s}$ as a typical sailplane cruise speed [14] [15]. As the stall condition angle of attack or higher values are not scope 
of this study, angle of attack range was used as changing between -6 to 6 degrees [1] [16].

\section{RESULTS AND DISCUSSION}

The results of numerical analyses performed on XFLR5 program at $10^{6}$ Reynolds number, which was calculated by using mean aerodynamic chord lengths of the models as the characteristic length, in terms of the span-wise local lift coefficients of the models were given in Figure 5. The model $1(\lambda=0.2)$ and model $2(\lambda=0.4)$ have their highest lift coefficients near the tip section of the wing. From model $3(\lambda=0.62)$ to model $6(\lambda=1.2)$, it was clearly seen that the maximum lift coefficient region has moved near the middle and root section of the wings.

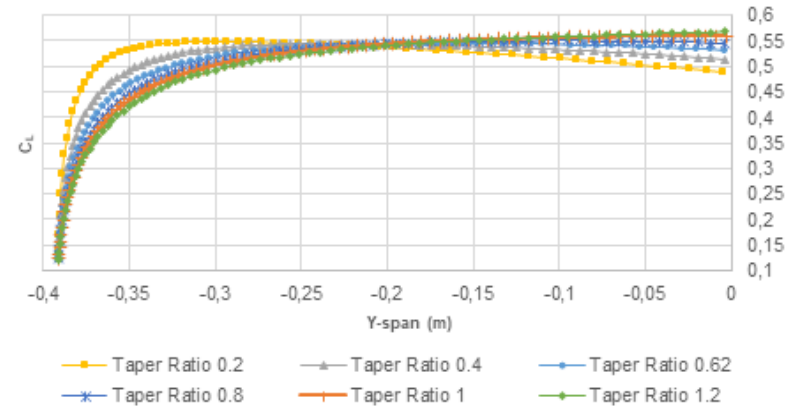

Figure 5: Local lift coefficient distributions of the models along their half spans

Span-wise lift distributions of each model were given together with their elliptical lift distributions in Figure 6. Firstly, the base model's lift distribution was compared with an experimental analysis result [17] and found in good agreement about its distribution. When the lift distributions of the models were compared with their elliptical distributions, near the root section of the wing, the lift distribution of the model $3(\lambda=0.62)$ was found to be most similar. Moreover, at the region from the middle to tip section of the wing, model $2(\lambda=0.4)$ was found to have most similar lift distribution.

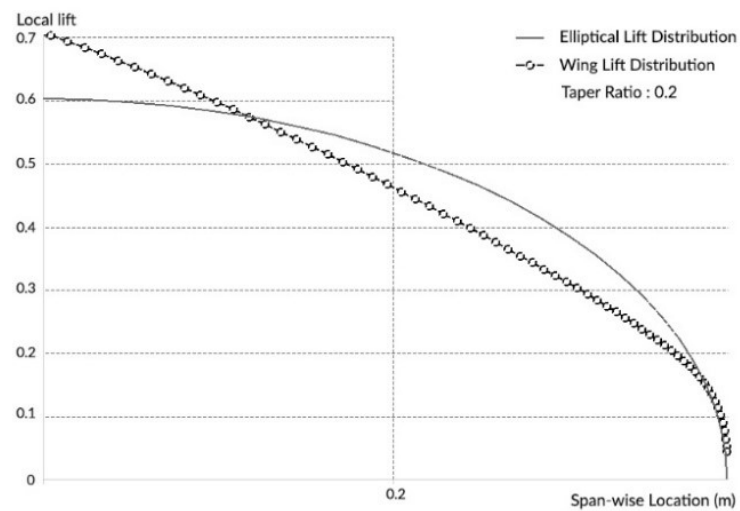

(a)

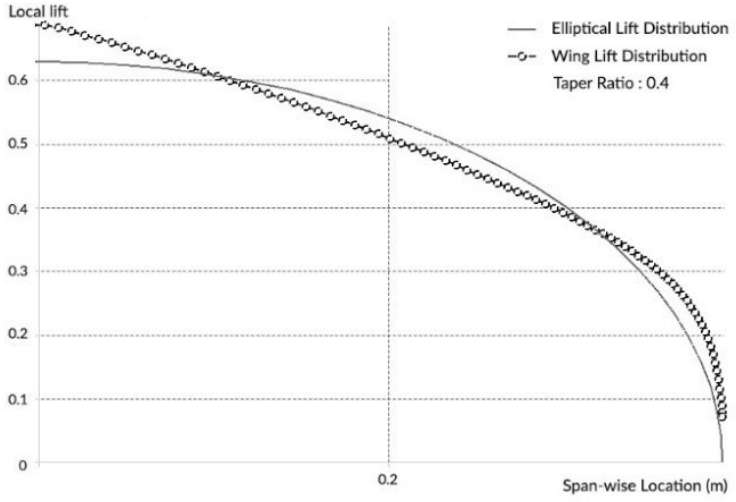

(b)

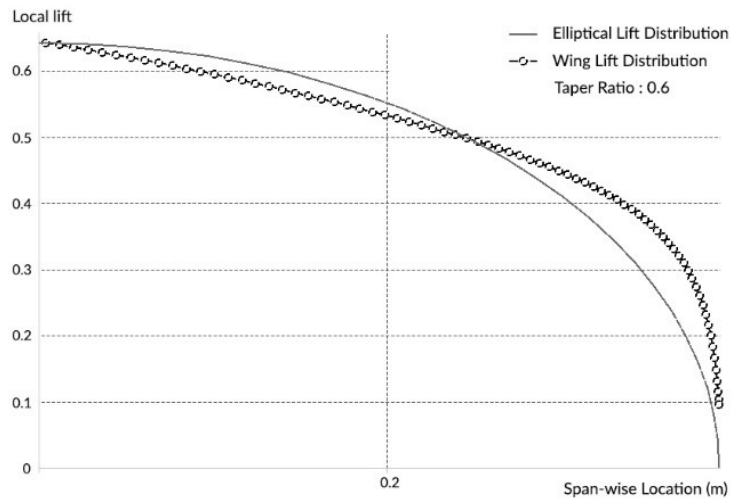

(c)

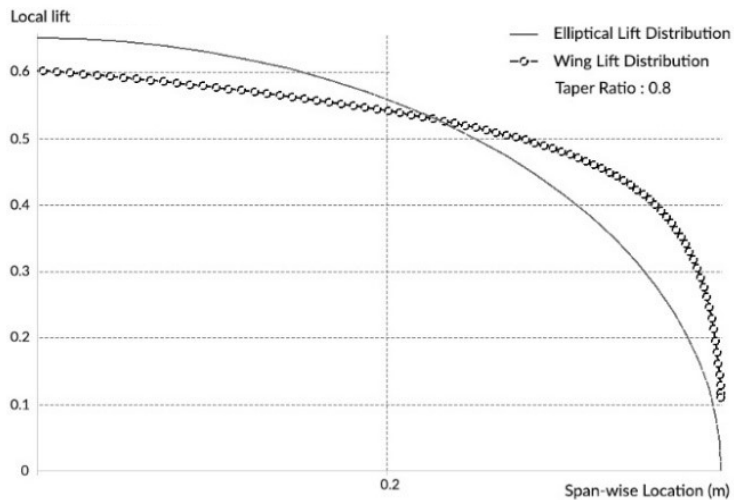

(d)

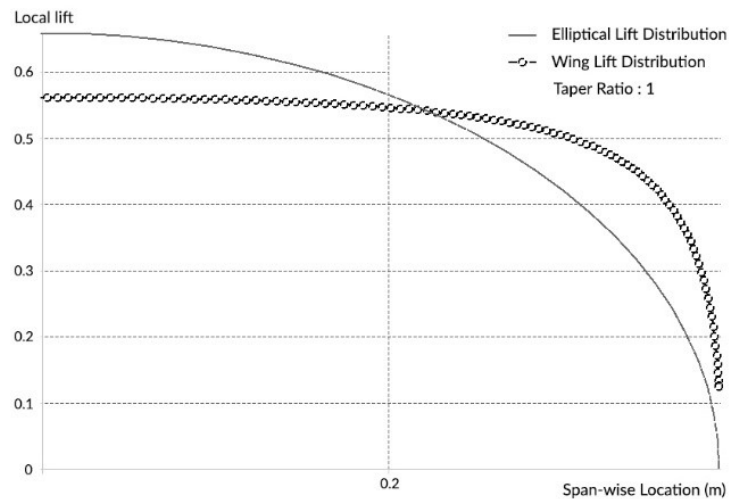

e) 


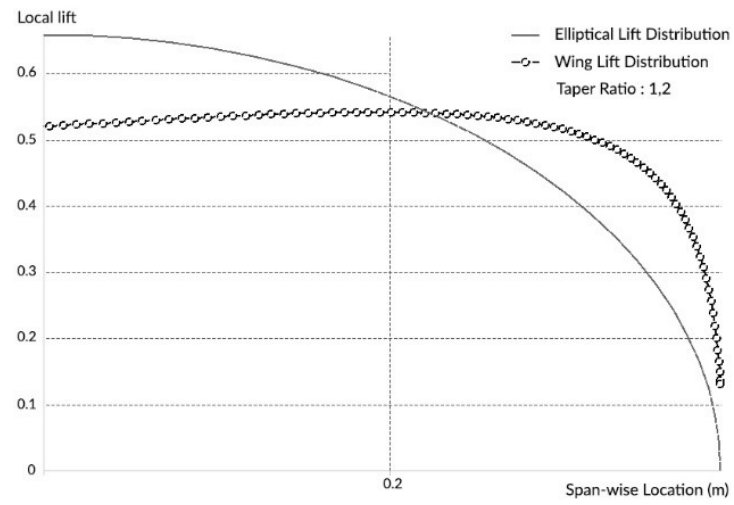

(f)

Figure 6: Span-wise lift distributions with elliptical lift distributions of the models; a) model 1, b) model 2 , c) model 3 , d) model 4 , e) model $5, \mathrm{f}$ ) model 6

Figure 7 and Figure 8 presents the numerical analysis results of the models in terms of the Oswald efficiency factor and induced drag parameter. As seen in the figures, at -6 degree angle of attack, Oswald efficiency factors of models were in order similar to their taper ratios. Between the angle of attacks -4 to 6 degrees, model $2(\lambda=0.4)$ has the highest and the model $6(\lambda=1.2)$ has the smallest Oswald efficiency factors. At zero angle of attack, model 1 (e=0.954), model $2(\mathrm{e}=0.977)$, model $3(\mathrm{e}=0.955)$ and model $4(\mathrm{e}=0.921)$ has Oswald efficiency factors higher than 0.9; model 5 (e=0.884) and model 6 ( $\mathrm{e}=0.851)$ has this values between 0.8 and 0.9 . As expected, investigation of induced drag parameter of each model also shows that, model $1(\lambda=0.2)$ and model 3 $(\lambda=0.62)$ has the same values $(\delta=0.05)$. Moreover, they have very similar Oswald efficiency factor changes at -6 to 6 degrees angles of attacks.

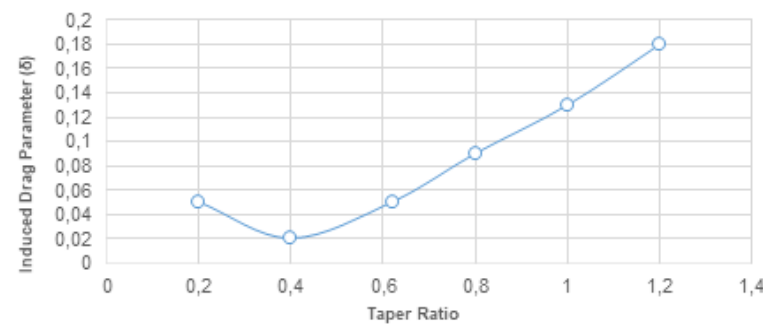

Figure 7: Induced drag parameters $(\delta)$ versus taper ratios of the models

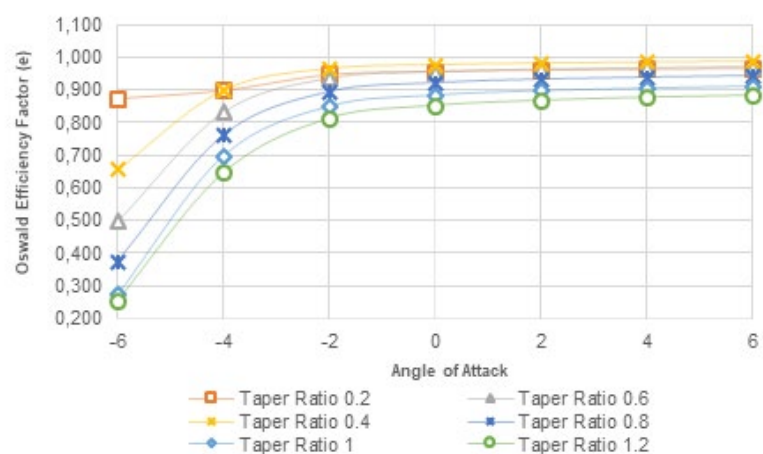

Figure 8: Oswald efficiency factors of the models versus angle of attack from -6 to 6 degrees

In Figure 9, the numerical results for induced drag coefficients changing with taper ratios of the models were given. As expected, the change of induced drag coefficient valu- es are in good agreement with the values of induced drag parameter. Model $2(\lambda=0.4)$ has the minimum $(=0.00543)$ and model $6(\lambda=1.2)$ has the maximum value $(=0.005886)$ of induced drag coefficient. Model 1, 3, 4 and 5 has induced drag values of $0.005449,0.005488,0.005622$ and 0.005806 , respectively.

Figure 10 shows the streamlines of each model at zero angle of attack at four times of chord length distance from the trailing edges of the wings. As seen, the minimum size of the wing tip vortices has seen at model $1(\lambda=0.2)$. The maximum size has seen on model $6(\lambda=1.2)$. Therefore, the sizes of wing tip vortices were found to be increased with the decrease in taper ratio.

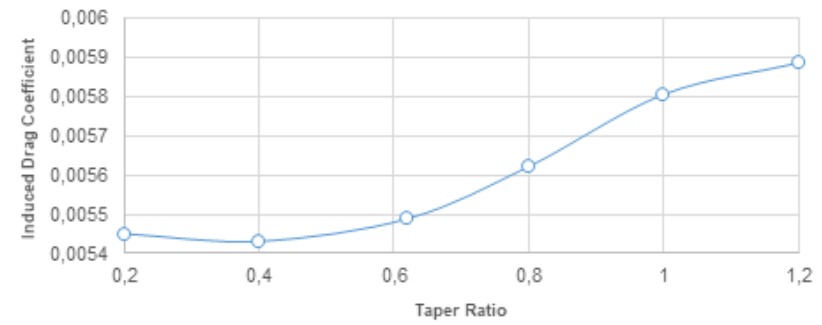

Figure 9: Induced drag coefficients versus taper ratios of the models

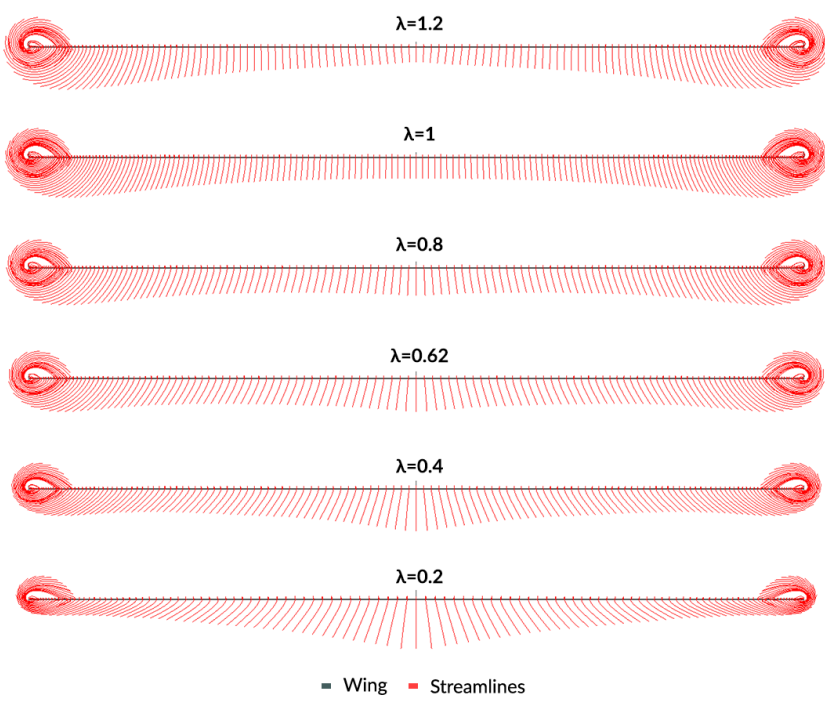

Figure 10: Front-view of streamlines and wing tip vortices of the models

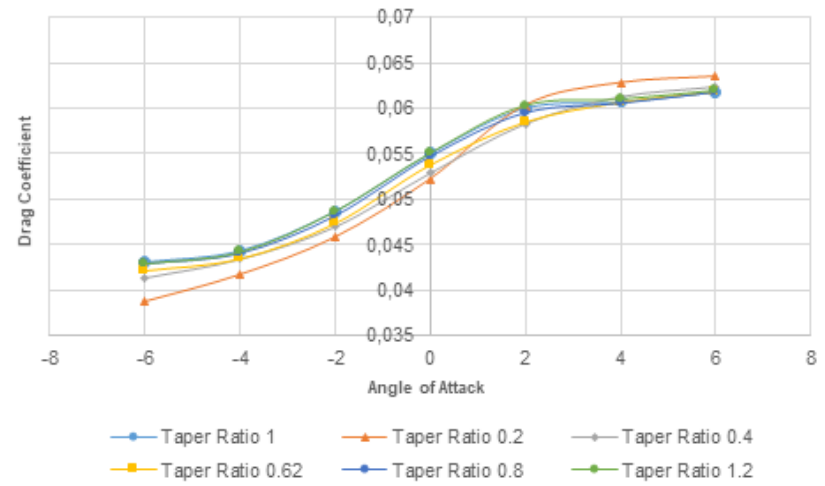

Figure 11: Drag coefficients of models versus angle of attack from -6 to 6 degrees

The change in total drag coefficient with angle of attack was given in Figure 11. It is seen from the figure that, model 1 
$(\lambda=0.2)$, has lower drag coefficients than other models at angle of attacks lower than 2 degree. On the contrary, it has higher values than other models at angle of attacks higher than 2 degree. Models 2, 3, 4, 5 and 6 was found to have similar changes between angle of attacks of -6 and 6 . At zero angle of attack, models 5 and 6 has approximately same drag coefficient values of 0.550 . Models 1, 2, 3 and 4 has drag coefficient values of $0.0521,0.0528,0.0538$ and 0.0546 , respectively.

\section{CONCLUSION}

In this study, with the aim of investigating effects of taper ratio on aircraft wing aerodynamic parameters, the high aspect ratio wings having different taper ratios were numerically analyzed. For the comparison of their aerodynamic parameters, a rectangular wing model was revised to five different models, which have different taper ratios, but have same wing area, aspect ratio and mean aerodynamic chord. Numerical analyses of the models were performed on XFLR5 program which uses Vortex Lattice Method. After the assessment of mesh accuracy of the program was done, all the models having taper ratios from 0.2 to 1.2 (with the increment of 0.2) were analyzed in terms of induced drag coefficient, Oswald efficiency factor, and lift coefficient together with span-wise lift distribution. According to numerical analysis results, it was obtained that, there is an optimum taper ratio value for a wing, which have minimum induced drag coefficient and maximum Oswald efficiency factor values. On the other hand, decreasing taper ratio so much was found to have the possibility of causing wing-tip stall due to higher local lift coefficients at the tip region of the wing. In addition, it was found that, size of wing-tip vortices were increased with the increase in taper ratio. As a future work, this study can expanded to experimental aerodynamic analyses and also, in terms of numerical analysis, taper ratio can investigated together with other wing geometric parameters such as aspect ratio, sweep angle and dihedral/ anhedral angles to observe their aerodynamic relationships.

\section{REFERENCES}

[1] Gudmundsson, S. (2013) General aviation aircraft design: Applied Methods and Procedures. Butterworth-Heinemann.

[2] Raymer, D.P. (2012). Aircraft design: a conceptual approach, AIAA Education Series. Reston, Virginia.

[3] Sadraey, M.H. (2012). Aircraft design: A systems engineering approach. John Wiley \& Sons.

[4] Bergmann, A., Huebner, A., and Loeser, T. (2008). Experimental and numerical research on the aerodynamics of unsteady moving aircraft. Progress in Aerospace Sciences, 2008. 44(2): p. 121-137. Doi:10.1016/j.paerosci.2007.10.006

[5] Zhang, P. F., Wang, J. J., Liu, Y., Wu, Z. (2009). Effect of taper ratio on aerodynamic performance of cropped nonslender delta wings. Journal of Aircraft, 46(1), 320-325.

[6] Bravo-Mosquera, P.D., Cerón-Muñoz, H.D., Díaz-Vázquez, G., Catalano, F.M. (2018). Conceptual design and CFD analysis of a new prototype of agricultural aircraft. Aerospace Science and Technology,
2018. 80: p. 156-176, Doi: 10.1016/j.ast.2018.07.014.

[7] Qin, N., Vavalle, A., Moigne A.L. (2005). Moigne, Spanwise Lift Distribution for Blended Wing Body Aircraft. Journal of aircraft, 2005. 42(2): p. 356-365. Doi:10.2514/1.4229.

[8] Lee, T., and Gerontakos, P. (2006). Effect of winglet dihedral on a tip vortex. Journal of Aircraft, 2006. 43(1): p. 117-124. Doi:10.2514/1.14052.

[9] Wakayama, S., and Kroo, I. (1995). Subsonic wing planform design using multidisciplinary optimization. Journal of Aircraft, 1995. 32(4): p. 746-753. Doi:10.2514/3.46786.

[10] Nelson, C. P. (1992). Effects of wing planform on HSCT off-design aerodynamics. in 10th Applied Aerodynamics Conference. 1992. Doi:10.2514/6.1992-269

[11] Chen, T. and J. Katz (2004). Induced Drag of High-Aspect Ratio Wings. in 42nd AIAA Aerospace Sciences Meeting and Exhibit. Doi:10.2514/6.2004-38

[12] Güzelbey, I.H., Eraslan, Y., and Doğru, M.H. (2018). Numerical Investigation of Different Airfoils at Low Reynolds Number in terms of Aerodynamic Performance of Sailplanes by using XFLR5. The Black Sea Journal of Sciences. 8(1): p. 47-65. Doi:10.31466/kfbd.423932

[13] Deperrois, A. (2009). XFLR5 Analysis of foils and wings operating at low Reynolds numbers. Guidelines for XFLR5.

[14] Thomas, F. and J. Milgram (1999). Fundamentals of sailplane design. Vol. 3. College Park Press, College Park, Maryland.

[15] Administration, F.A. (2007). Glider Flying Handbook. Skyhorse Publishing Inc.

[16] Frati, S. (1946). The Glider. Editore Ulrico Hoepli Milano, Milan, Italy.

[17] Diederich, F. W., Zlotnick, M. (1953). Calculated spanwise lift distributions and aerodynamic influence coefficients for unswept wings in subsonic flow. NASA Technical Note 3014.

[18] Zhang, P. F., Wang, J. J., Liu, Y., \& Wu, Z. (2009). Effect of taper ratio on aerodynamic performance of cropped nonslender delta wings. Journal of Aircraft, 46(1), 320-325. 